\title{
Research Status and Development Trend of Automatic Quantitative Technology of Small-Caliber Gun Propellant
}

\author{
D. Xu, B.H. Han, Z.G. Cheng, W.H. He \\ Mechanical Engineering College, Shijiazhuang, China
}

\begin{abstract}
The small-caliber artillery test ammunition is various, high precision in shooting range. But the Chinese domestic is still in the method of manual quantitative. In order to improve the accuracy and speed of quantity, researched common volumetric quantitative technology, weighing quantitative technology and combined quantitative technology, analyzed their advantages and disadvantages. Use for reference from quantitative technology of food, medical and other industries, applied to the ordnance propellant quantitative technology. On the basis of the existing theory, speculated on future studies of high precision automatic quantitative technology of small-caliber gun propellant is based on dynamic weighing, developing to the intelligent, integrate, fully automated direction.
\end{abstract}

KEYWORD: propellant; automatic quantitative; dynamic weighing; accurate.

\section{INTRODUCTION}

The rapid development of science and technology of the modern battlefield, endless variety of weapon equipment. To meet the high quality of modern warfare, test is essential to weapon equipment's performance. Weapon test site is committed to equipment performance testing, test of gun weapon used to experimental ammunition. Among them, it is various of small-caliber experimental ammunition, including $23 \mathrm{~mm}, 25 \mathrm{~mm}, 31 \mathrm{~mm}, 37 \mathrm{~mm}$ and $57 \mathrm{~mm}$ caliber. The assembly line is consisted of fire transferring tube screwed, supporting chamber paper loaded, propellant quantified and loaded into the cartridge, decoppering agent and paper cover loaded, combined with ammunition and other processes (Ma Yunfu,2009), as figure 1 .

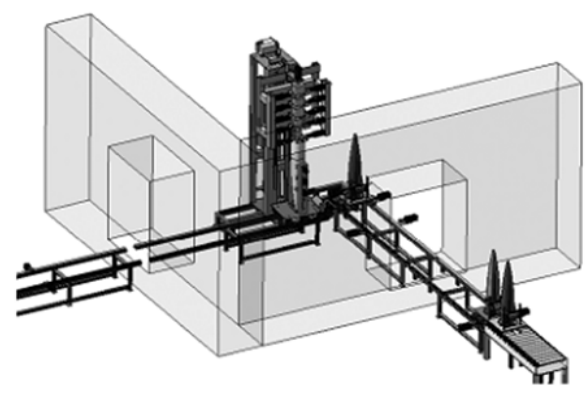

Figure 1. The automatic device for ammunition loading.
The propellant automatic filling technology is mainly automatic quantitative and transfers it into the cartridge, which is the key of assembly. Propellant is the ultimate expression of projectile energy, quality and weight of the propellant has a directly affect the performance of the shell. Therefore, quantitative accuracy requirement of the device is particularly high, the highest quantitative accuracy requires to achieve $0.1 \mathrm{~g}$.

Currently, test ammunition's propellant loading process is still the traditional manual method in test base, through artificial control balance or electronic scales weigh propellant to the required accuracy. Gunpowder is a kind of inflammable and explosive substance. The performance is instability, extremely dangerous, causing the loss of personal and property. Therefore, the research of the automatic loading system, on one hand can avoid harm, brought by propellant to the health of workers, on the other hand, artificial filling high is labor-intensive, slow speed. It is difficult to adapt to high speed of the modern battlefield situation. Using the propellant automatic filling device can be fully automated control and improve production efficiency. Automatic loading of propellant also requires attention to the accuracy and speed. 


\section{COMMON PROPELLANT AUTOMATIC QUANTITATIVE TECHNOLOGIES}

Automatic quantity is the key technology of automatic loading. Propellant on the demand for precision is high, but also is taken into account the quantitative rate, so use fast-charge and fine-charge. The fast-charge refers to fill the propellant to a critical value quickly, then to fine-charge, until it reaches the quantitative requirements. Meanwhile, control the loading density of the propellant.

Propellant of test base usually is granular. According to different quantitative methods, the quantitative analysis of granular materials can be divided into volumetric quantitative technology, weighing quantitative technology and combined quantitative technology. Three methods have their advantages and disadvantages, suitable for different occasions.

The volumetric quantitative technology is a traditional quantitative method, has been extensively used in the community, which uses a fixed volume of the device to fill propellant. This device has the advantages of low cost, simple structure and fast filling speed. The volumetric quantitative device is generally used in small-caliber gun propellant. Small-caliber gun propellant is mostly in the form of pellets, small size and products quickly. However, the accuracy of the volumetric quantitative technology is easy to be influenced by the physical properties such as uniformity, adsorption and bulk density. At present, in order to improve the volumetric quantitative precision, using the volume adjustable mechanical device, and propellant is mostly uniform size, excellent fluidity. Its weighing accuracy can reach about 1\% 3\% (Xie Yu, 2013).

The weighing quantitative technology is also a traditional way to weigh. Through weighing to determine the measure of propellant, so as to fill. The weighing quantitative technology in accordance with the mechanical structure is divided into electronic weighing equipment, mechanical weighing equipment and combined weighing equipment. Compared with the volumetric weighing technology, the structure is complex, the quantitative accuracy is high, but the speed is slow. Currently, the weighing quantitative techniques, for both speed and accuracy of feeding, mostly using multilevel feeding. Multilevel feeding refers to firstly through the fast-feeder to add the most of the target weight of propellant to the hopper, usually $90 \% \sim 95 \%$ of the total. The residual material added into the hopper slowly by fine-feeder until it reaches requirements of the target weight range. Quantitative accuracy of the weighing quantitative technology can reach 1\% (Xie Yu, 2013).

The combined quantitative technology is a kind of advanced new technology developed on the basis of the traditional volumetric quantitative technology and weighing quantitative technology. Tian Pengzhi put forward the concept of mixed quantitative, and used in the packaging machine (Tian Pengzhi,
1991). The combined quantitative technology combines the advantages of the volumetric quantitative technology fast speed and the weighing quantitative technology high precision. The combined quantitative techniques uses multilevel feeding mode, with fast-feeder device and volumetric weighing method to fill a lot of material into the hopper quickly. It has the same accuracy as the fast filling of the weighing quantitative technology, achieves $90 \% \sim 95 \%$ of the target weight. After weighing device is stable, calculates the amount of material required for fine-feeder, and convert it into fine-feeder filling propellant flow and time by control system. Then fills the propellant controlled by controller. Fast-feeder device and finefeeder device use the volumetric quantitative technology, but fine-feeder set a small amount of material. So the volumetric quantitative technology error is reduced and increase the accuracy of quantitative filling. In addition, after the fine-feeding, need not to weigh, so enhance the speed of filling. Quantitative accuracy of combined quantitative technology can reach $1 \%$. If there is a requirement to further improve the quantitative accuracy, can be appropriated to reduce the filling speed. After the fine-feeding and weighing, again the second filling by the finefeeder and control hopper one-time add propellant to cartridge at last, such quantitative precision can reach $0.5 \%$. The combined quantitative technology processes are shown in figure 2.

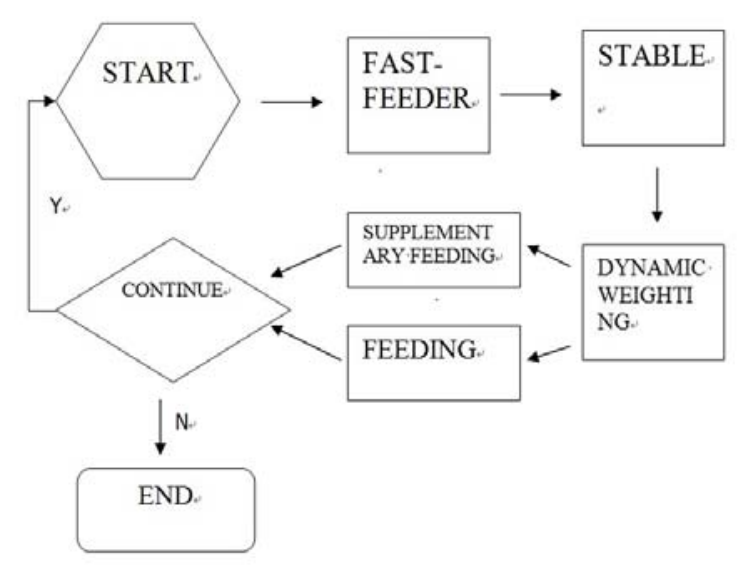

Figure 2. The combined quantitative technology processes.

Above all, the volumetric quantitative technology is fast, but low precision. The accuracy of the weighing quantitative technology is high, but slow speed. The combination quantitative technology improves the precision, and the speed is fast, but the structure is complex, and the cost is high. Nevertheless it is difficult to realize the control of granule by the combined quantitative technology at present. 


\section{CONCLUSION}

The automatic quantitative theory is not perfect. In order to improve the existing automatic quantitative technologies, except find a suitable quantitative method, it can also improve quantitative process and improved control method to improve the quantitative accuracy and speed. Ma Xing aimed at the granular propellant, putted forward a new dynamic weighing method (Ma Xing, 2012). It referred to material was still on the bench vibration, could get accurate weight, filled and weighed at the same time. Dynamic weighing method could improve efficiency and reduce the labor intensity of workers. The key to achieve fast and accurate dynamic weighing is how to get the dynamic response signal in the measurement process through the software and hardware processing, as figure 3 .

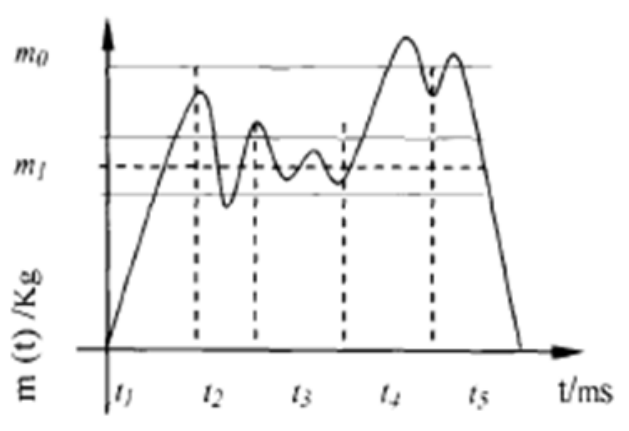

Figure 3. Dynamic response signal.

At present, in most cases, dynamic measurement uses the same pressure sensor as the static weighing to detect the weight step response signal. This kind of sensor response speed is slow, overshoot is large. Usually, after response signals attenuate to a certain extent, and then measure and process, so that the speed of measurement slow down. Therefore, how to improve the testing speed is the problem to be solved in the research of dynamic weighing technology under the premise of ensuring the accuracy of inspection. Through the design of compensating element, improve the sensor precision, reduce overshoot, reduce the weighing sensor stable time, improve the dynamic weighing accuracy and speed. The compensating element via dampers and other compensation components to improve the fast stability of weighing step response signals, but also can use digital compensation, through digital processing adaptive dynamic compensation method and improve the weighing system adaptability for quality changes of the weighing object (Zhang Xiliang, 2008).

Guo Xiangjun applied PLC and touch screen to compose control system on the quantitative automatic weighing packaging machine to replace the traditional weighing instrument control (Guo Xiangjun,
2007). She found the system can control the quantity of input and output effectively, but also easy to set up and modify the system parameters, improve filling accuracy and speed, meanwhile save cost.

Lu Ye, Yuan Meng, through contrast tests, for mixed automatic quantitative feeder, applied the principle of fuzzy PID control (Lu Ye, 2008). They found it can improve quantitative accuracy greatly, and effective. Lin Chunmei, Zhang Dejun, also applied fuzzy control technology with high precision automatic feeding system, this research realized intelligent control of the vibration frequency and amplitude, according to the discharging speed change (Lin Chunmei, 2015).

With the development of PLC, SCM and other controllers and vibration sensor, the automatic quantitative technology of small-caliber gun propellant is developing to the direction of digitization, integration and intelligence.

\section{ACKNOWLEDGEMENTS}

This work is supported by the Test Technology of General Armament Department.

\section{REFERENCES}

Guo, X.J. \& Li, Z.L. (ed.) 2007. The application of PLC in the weighing type automatic quantitative packaging machine, Micro Computer Information, (09): 57-58, 80.

Han, X.B. 2013 Research and development of automatic quantitative measurement system in packaging machinery. Beijing: Beijing University of Posts and Telecommunications.

Lu, Y \& Yuan, M. 2008. Study on the application of fuzzy PID control in hybrid automatic quantitative feeder, Packaging Engineering, (06): 14-16, 20.

Lin, C.M \& Zhang, D.J. 2015. High precision powder feeding system based on fuzzy control, Mechanical Management and Development, (01): 14-16.

Ma, Y.F. 2009. The present situation and development countermeasure of ammunition assembly technology in our country, Ordnance Industry Automation, 29(8): 1-3, 14.

Ma, X, \& Zhang, B \& Liang, X.H.(ed.) 2012. Dynamic automatic weighing technology, Ordnance Industry Automation, 31(4): 69-70, 74.

Tian, P.Z. 1991. Application of hybrid quantitative method in packaging machine, China Packaging Industry, (1).

Xie Yu. 2013. Research on key technologies of automatic quantitative propellant. Shijiazhuang: Ordnance Engineering College.

Zhu, Z \& Zhang, B (ed.) 2015. The comparison and development analysis of automatic weighing technology of propellant, Machinery and Electronics, (4): 75-78.

Zhang, X.L. 2008. Automatic quantitative mechanism and system development of powder materials. Zhenjiang: Jiangsu University. 